\title{
CLIMA URBANO E SAÚDE: ELEMENTOS CLIMÁTICOS E DOENÇAS RESPIRATÓRIAS OBSERVADAS NO MUNICÍPIO DE BELO HORIZONTE (MG) ENTRE 2013 E 2014
}

\author{
SALES, Denise Marques - denise.marques.sales@gmail.com \\ Universidade Federal de Minas Gerais / UFMG \\ ASSIS, Wellignton Lopes- assisw@gmail.com \\ Universidade Federal de Minas Gerais / UFMG \\ FONSECA, Bráulio Magalhães-brauliomagalhaes@gmail.com \\ Universidade Federal de Minas Gerais / UFMG
}

\begin{abstract}
RESUMO: No município de Belo Horizonte (MG) são registrados todos os anos um número elevado de ocorrência de doenças respiratórias, constituindo em uma das principais causas de internação no Sistema Único de Saúde. Diante desse cenário, este estudo teve como objetivo identificar a relação entre os elementos climáticos e a ocorrência de doenças respiratórias na capital mineira. Para atingir o objetivo proposto, foram utilizados registros mensais de cinco estações meteorológicas pertencentes à Fundação Estadual do Meio Ambiente e de quatro estações meteorológicas do Instituto Nacional de Meteorologia, localizadas dentro do município e/ou próximas ao limite municipal. Foram analisados dados sobre a temperatura do ar, umidade relativa, precipitação, particulados (PM10) e monóxido de carbono para os anos de 2013 e 2014. Os sistemas atmosféricos atuantes no período foram identificados utilizando-se cartas sinóticas, disponibilizadas pela Diretoria de Hidrografia e Navegação da Marinha do Brasil, e imagens de satélite, disponíveis na página do Instituto Nacional de Pesquisas Espaciais. As informações relacionadas às doenças respiratórias foram disponibilizadas pela Secretaria Municipal de Saúde de Belo Horizonte. Nesse banco de dados consta o número de atendimentos em unidades de saúde pública registradas com o Código Internacional de Doenças (CID) 10, de J00 a J99, para os sexos feminino e masculino de crianças de 0 a 5 anos. A interpolação, a análise multicritério e espacialização dos dados foram feitas no software ArcGis 10.3 e em planilha eletrônica do Microsoft Excel. Os resultados mostraram tendências sazonais nos picos de atendimentos nas unidades de saúde, principalmente para o mês de abril. A partir desse mês o volume diário de precipitação é reduzido e verifica-se um aumento gradativo na quantidade de particulados e CO na atmosfera belo-horizontina. É um período em que são registradas elevadas amplitudes térmicas diárias em função da participação de sistemas atmosféricos estáveis, principalmente do Anticiclone Subtropical do Atlântico Sul e da Massa Polar Atlântica. Existem evidencias de que as crianças sofrem mais nessas condições de tempo meteorológico, aumentando os riscos a infecções por vírus e bactérias no sistema respiratório.
\end{abstract}

PALAVRAS-ChAVE: Elementos Climáticos. Ambiente Urbano. Doenças Respiratórias.

URBAN CLIMATE AND HEALTH: CLIMATIC ELEMENTS AND RESPIRATORY DISEASES OBSEVERD IN THE CITY OF BELO HORIZONTE (MG) BETWEEN 2013 AND 2014

\begin{abstract}
In the municipality of Belo Horizonte (MG), a high number of respiratory diseases is recorded every year, constituting one of the main causes of hospitalization in the Unique Health System. Given this scenario, this study had as aim to identify the relationship between climatic elements and the occurrence of respiratory diseases in the capital of Minas Gerais. To reach the proposed aim, records of five meteorological stations belonging to State Foundation for the Environment and four weather stations of the National Meteorology Institute were used, which are located within the municipality and / or near the municipal boundary. Data about air temperature, relative humidity, precipitation, particulates (MP10) and carbon monoxide for the years 2013 and 2014 were analyzed. The atmospheric systems operating in the period were identified using the synoptic charts provided by Hydrography and Navigation Board from the Brazilian
\end{abstract}


Navy and the satellite images available at the National Institute for Space Research. The information related to respiratory diseases was made available by Belo Horizonte Department of Health. This database includes the number of visits to public health units registered with International Code of Diseases (ICD) 10, from J00 to J99, for the female and male children from 0 to 5 years old. The interpolation, multicriteria analysis and data spatialization were done in ArcGis 10.3 software and through the Microsoft Excel spreadsheet. The results showed seasonal trends in the attendance peaks in the health units, especially on April. From that month the daily precipitation volume is reduced and there is a gradual increase in the amount of particulates and $\mathrm{CO}$ in the municipal atmosphere. It is also a period in which high daily thermal amplitudes are registered due to the participation of stable atmospheric systems, mainly the Atlantic South Anticyclone Subtropical and Atlantic Polar Air Mass. There is evidence that children suffer more under these weather conditions, increasing the risk of infection by viruses and bacteria in the respiratory system

KEYWORDS: Climatic Elements. Urban Environment. Respiratory Diseases.

\section{CLIMA URBANO Y SALUD: ELEMENTOS CLIMÁTICOS Y ENFERMEDADES RESPIRATORIAS OBSERVADAS EN EL MUNICIPIO DE BELO HORIZONTE (MG) ENTRE 2013 Y 2014}

RESUMEN: En el municipio de Belo Horizonte (MG) se registran todos los años un número elevado de ocurrencia de enfermedades respiratorias, constituyendo una de las principales causas de internación en el Sistema Único de Salud (SUS). Ante este escenario, este estudio tuvo como objetivo identificar la relación entre los elementos climáticos y la ocurrencia de enfermedades respiratorias en el municipio de Belo Horizonte. Para alcanzar el objetivo propuesto, se utilizaron registros mensuales de cinco estaciones meteorológicas pertenecientes a la Fundación Estadual del Medio Ambiente y de cuatro estaciones meteorológicas del Instituto Nacional de Meteorología, ubicadas dentro del municipio y / o cercanas al límite municipal. Se analizaron datos sobre la temperatura del aire, humedad relativa, precipitación, partículas (PM10) y monóxido de carbono para los años 2013 y 2014. Los sistemas atmosféricos actuantes en el período fueron identificados utilizando las cartas sinópticas, disponibles por el Centro de Previsión de Tiempo y Estudios Climáticos y por la Dirección de Hidrografía y Navegación de la Marina de Brasil, e imágenes de satélite, disponibles en la página del Instituto Nacional de Investigaciones Espaciales. Las informaciones relacionadas con las enfermedades respiratorias fueron puestas a disposición por la Secretaría Municipal de Salud de Belo Horizonte, a través de la Asesoría de Tecnología e Información en Salud. En este banco de datos constan el número de atendimientos en unidades de salud pública registradas con el Código Internacional de Enfermedades (CID 10), de J00 a J99, para los géneros femenino y masculino de niños de 0 a 5 años. La interpolación, el análisis multicriterio y la espacialización de los datos se realizaron en el software ArcGis 10.3 y en la hoja de cálculo de Microsoft Excel. Los resultados mostraron tendencias estacionales en los picos de atención en las unidades de salud, principalmente para el mes de abril. A partir de ese mes el volumen diario de precipitación es reducido y se verifica un aumento gradual en la cantidad de partículas y $\mathrm{CO}$ en la atmósfera de la ciudad objeto. También es un período en que se registran elevadas amplitudes térmicas diarias en función de la participación de sistemas atmosféricos estables, principalmente del Anticiclón Subtropical del Atlántico Sur y de la Masa Polar Atlántica. Existen evidencias de que los niños sufren más en esas condiciones de tiempo, aumentando los riesgos a las infecciones por virus y bacterias en el sistema respiratorio.

PALABRAS CLAVE: Elementos Climáticos. Ambiente Urbano. Enfermedades Respiratorias.

\section{INTRODUÇÃO}

Atualmente é perceptível a complexidade envolvendo articulações entre clima, tempo e saúde. Em ambientes de clima tropical, fenômenos como elevada amplitude térmica diária, ondas de calor, chuvas intensas, inundações 
repentinas, vendavais, baixa umidade do ar e concentração de poluentes na atmosfera, afetam diretamente a população.

O conjunto das relações entre elementos climáticos e as populações, geram impactos potencializados em escalas regional e local, em função de diferentes graus de vulnerabilidade e capacidade adaptativa das populações que ali vivem. Visando entender melhor essas especificidades e com o objetivo de buscar soluções para os riscos à saúde respiratória, a Organização Mundial da Saúde (OMS), a Organização Pan-Americana de Saúde (OPAS) e o Ministério da Saúde (MS), têm elaborado estudos e medidas importantes nos últimos anos trazendo discussões de forma interdisciplinar.

Dado os efeitos da poluição do ar sobre a saúde humana, a OMS publicou o Air quality guidelines for particulate matter, ozone, nitrogen dioxide and sulfur dioxide (WHO, 2005). Nesse documento constam diretrizes para promover a qualidade do ar e reduzir os efeitos da poluição atmosférica na saúde, o objetivo é auxiliar os formuladores de políticas em relação à gestão da qualidade do ar em diferentes partes do mundo.

A OMS alerta sobre o impacto das doenças respiratórias crônicas (DRC) e os fatores de risco em documentos que abordam estudos de organizações nacionais e internacionais (WHO, 2005; WHO, 2007). Os meios de prevenção e tratamento dessas enfermidades foram publicados no Preventing Chronic Diseases, a vital investment (WHO , 2005) e na Global Surveillance, Prevention and Control of Chronic Respiratory Diseases, a Comprehensive Approach 2 (WHO, 2007).

Outro marco relevante para esta temática foi o plano de ação Global Alliance against chronic Respiratory Diseases (GARD) e a criação do Programa Nacional para as Doenças Respiratórias (ANTUNES; BÁRBARA; GOMES; 2013) publicado em 2013, que menciona que:

o enorme impacto e sofrimento humano resultante das DRC foi reconhecido pela 53a Assembleia Mundial da Saúde (Resolução WHA53.17, maio de 2000), que levou a OMS, em 2000, a darIhes prioridade no âmbito da prevenção e controle das doenças não transmissíveis, pressionando os estados membros a implementar medidas que visem esse objetivo, com especial ênfase nos países em desenvolvimento e em outras populações carentes [adaptado pelo autor]. (ANTUNES; BÁRBARA; GOMES; 2013, p.5)

As pesquisas acadêmicas relacionadas à temática clima e saúde ganharam maior notoriedade no Brasil a partir da década de 90 . Duchiade (1992) realiza uma revisão bibliográfica importante e um resgate histórico acerca de como eram tratadas as variáveis ambientais, chamadas anteriormente de ecológicas, e os agravos às doenças respiratórias. O artigo ressalta uma preocupação inicial do efeito nocivo e prejudicial causado pelo tabagismo e resgata alguns estudos internacionais das décadas de 70 e 80 que passaram a investigar o papel dos materiais particulados na ocorrência das doenças respiratórias.

A autora ressalta ainda que não há dúvidas acerca dos efeitos nocivos de episódios agudos da poluição do ar intensa sobre as vias respiratórias, levando 
ao agravamento de sintomas em indivíduos vulneráveis portadores de problemas respiratórios crônicos e até mesmo à morte (DUCHIADE, 1992).

Santos (2007) afirma que estudos realizados em todo o mundo, especialmente nos últimos 50 anos, apontam para associações positivas entre a exposição aos contaminantes do ar atmosférico, sobretudo nas áreas urbanas, e os efeitos adversos à saúde. A OPAS (2008) declara que o clima, entre outros fatores, pode suscitar a manifestação de determinadas doenças à saúde através de seus elementos (temperatura, umidade do ar, precipitação, pressão atmosférica e ventos), que interferem no bem-estar das pessoas.

Os agravos à saúde respiratória ocorrem principalmente nos grandes centros urbanos e a metrópole belo-horizontina não foge à regra. As doenças respiratórias são um problema para a saúde pública, ocupando posição de destaque entre as principais causas de internação no Sistema Único de Saúde (SUS) da capital mineira. Diante da perspectiva de possíveis relações entre clima e saúde o objetivo deste trabalho foi verificar a relação entre os elementos climáticos e a ocorrência de doenças respiratórias em crianças de 0 a 5 anos de idade no município de Belo Horizonte.

Segundo o IBGE (2010), a capital do Estado de Minas Gerais possui um território de $331.401 \mathrm{~km}^{2}$ e uma população de 2.375 .151 habitantes, estimativa de cerca de 2,5 milhões de habitantes para o ano de 2018, com aproximadamente $7,5 \mathrm{mil}$ hab/ $\mathrm{Km}^{2}$. O município apresenta características fisiográficas e socioambientais que contribuem para uma diversidade de topoclimas e mesoclimas e podem potencializar a ocorrência das doenças do aparelho respiratório.

Sabendo que as implicações do clima e do tempo atmosférico sobre a saúde humana ainda carecem de mais estudos, esse trabalho busca evidências que respondam às perguntas "Como?", "Onde?" e "Quando?" as doenças respiratórias ocorrem no município. Espera-se contribuir para uma melhor compreensão das condições de vulnerabilidade populacional em ambientes urbanos relacionadas à saúde pública, o que permitirá a aplicação e/ou elaboração de políticas públicas mais eficientes no município.

Sabe-se que a eficiência dos programas de saúde pública depende, visivelmente, de uma boa gestão territorial e que de acordo com Santos (1998), deve garantir o acesso aos bens e serviços básicos para uma boa qualidade de vida.

\section{MATERIAIS E MÉTODOS}

As informações relacionadas às doenças respiratórias foram disponibilizadas pela Secretaria Municipal de Saúde de Belo Horizonte (SMSA) através da Assessoria de Tecnologia e Informação em Saúde (ASTIS). Nesse banco de dados consta o número de atendimentos em unidades de saúde pública registradas com o CID 10 (Código Internacional de Doenças) de 100 a 199 (Doenças Respiratórias), para os sexos feminino e masculino de crianças de 0 a 5 anos (faixa etária mais vulnerável) nos anos de 2013 e 2014. Ressalta-se que esses dados foram disponibilizados a partir da aprovação do Comitê de Ética em Pesquisa - CAAE 81047717.9.0000.5140, e estão correlacionados com 173 equipamentos de saúde do SUS distribuídos ao longo dos nove (9) distritos sanitários (FIG.1). 
Para os dados climáticos, foram utilizados os registros diários do mês de abril dos anos de 2013 e 2014 de cinco estações meteorológicas pertencentes à Fundação Estadual do Meio Ambiente (FEAM) e de quatro estações meteorológicas do Instituto Nacional de Meteorologia (INMET), localizadas dentro do município e/ou próximas ao limite municipal (FIG.1). A escolha do mês de abril para este estudo foi em função do maior número de ocorrências das doenças respiratórias na população alvo.

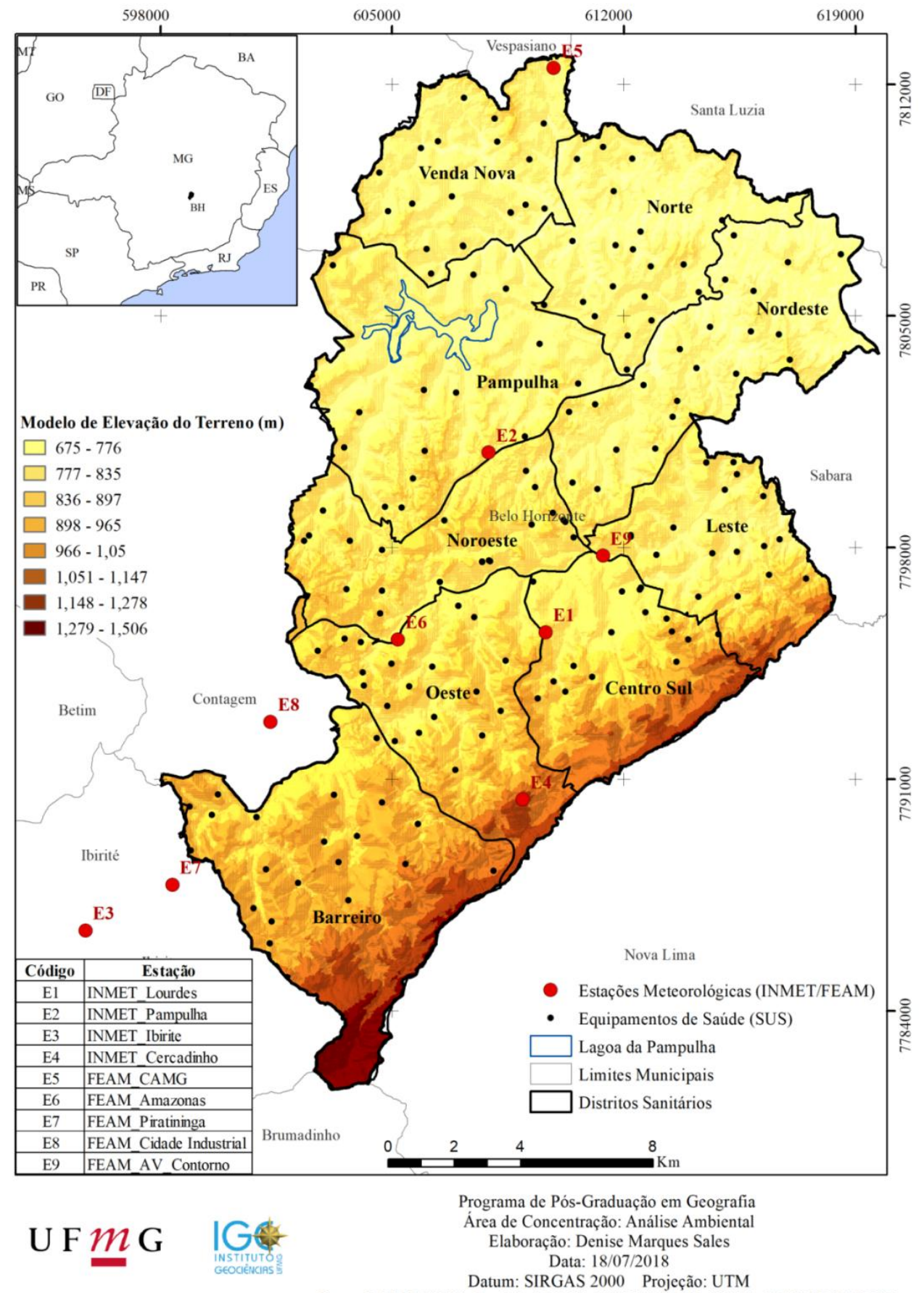

Fonte: FEAM (2017), Prodabel (2017), INMET (2018), SMSA (2017) IBGE(2010)

Figura 1 - Localização das estações meteorológicas e equipamentos de saúde no município de Belo Horizonte. 
Os elementos climáticos analisados foram: temperatura do ar, umidade relativa, precipitação; particulados (PM10) e monóxido de carbono (CO) para os anos de 2013 e 2014. Ao avaliar essas variáveis, identificou-se algumas falhas nos registros mensais. Como alternativas para preenchimento dos dados faltantes, foram testados dois métodos: interpolação e regressão linear. A interpolação e espacialização foram realizadas no software ArcGis 10.3 a partir da ferramenta Geostatistical Analyst e a regressão linear por meio da planilha eletrônica Microsoft Excel.

Os interpoladores testados foram Inverse Distance Weighting (IDW), Radial Basis Functions (RBF), Krigagem e Co_Krigagem, para a média mensal de temperatura, umidade, PM10 e CO e a precipitação acumulada mensal. Para a escolha do método de interpolação mais adequado, utilizou-se os valores de mean (mais próximo de zero melhor) e rms (quanto mais próximo de um melhor). Ressalta-se que as interpolações cujos modelos produziram homogeneização na espacialização, ou seja, mesmo valor para todas as falhas, foram excluídos.

Adotou-se o RBF como interpolador mais ajustado e adequado para o preenchimento das falhas nos registros de umidade relativa e precipitação, os resultados podem ser observados nas figuras 6 e 8 . A escolha de interpoladores para dados climáticos não é uma tarefa simples e o resultado final não depende apenas do interpolador. O processo de interpolação exige conhecimento acerca da natureza do dado a ser interpolado e da distribuição espacial das amostras (PERIN et al., 2015).

A regressão linear foi realizada através de ferramenta do banco estatístico do software Excel, tomando-se como base a equação 1 a seguir (SANTOS et al.,2015)

$$
\mathbf{T}=\beta_{0}+\beta_{1} Y+\beta_{2} X+\beta_{3} A L T
$$

Equação 1: Regressão Linear para preenchimento de falha, temperatura média.

Em que,

T: Temperatura do ar $\left({ }^{\circ} \mathrm{C}\right)$;

$\beta_{0}$ : Constante de regressão;

Y: Coordenada Geográfica Y (latitude);

X: Coordenada Geográfica X (longitude);

ALT: Altitude (m); e

$\beta_{1}, \beta_{2}$,e $\beta_{3}$ : Coeficientes de regressão para as variáveis $\mathrm{Y}, \mathrm{X}$ e ALT

Depois das falhas identificadas, realizou-se uma regressão linear tomando como base a relação entre a variável dependente (valores de temperatura) e independentes representados pela altitude (em metros) e as demais coordenadas UTM para Longitude (X) e Latitude (Y). Ao final foi gerado um resumo estatístico com os valores correspondentes para aplicação da equação 1 , calculada em cada célula com as falhas de dados.

Conforme ilustra da tabela 1 , os valores calculados estão em negrito e destacados em cinza. Importante observar o erro quadrado $\left(r^{2}\right)$ gerado na regressão com os valores existentes (sem considerar a falha) antes da aplicação da equação e posterior ao preenchimento da falha, em que o valor do $r^{2}$ mais adequado seja o mais próximo de 1 . Nesse caso, observou-se valores do $r^{2}$ 
muito próximos de um (1) para as variáveis temperatura, PM10 e CO. Isso indica um alto grau percentual da qualidade e ajuste do modelo. A partir desses resultados, os valores estimados por esse método foram os adotados.

Tabela 1 - Resultado com os preenchimentos das falhas para a variável temperatura média a partir da regressão linear.

\begin{tabular}{|c|c|c|c|c|c|c|c|c|c|c|c|c|c|c|c|c|c|}
\hline \multicolumn{18}{|c|}{ Temperatura Média $\left({ }^{\circ} \mathrm{C}\right)$ - Ano 2013 - Regressão Linear } \\
\hline Código & Estações & $\begin{array}{l}\text { Longitude } \\
(\mathrm{x})\end{array}$ & $\begin{array}{l}\text { Latitude } \\
\text { (y) }\end{array}$ & $\begin{array}{l}\text { Altitude } \\
(\mathrm{m})\end{array}$ & Jan & Fev & Mar & Abr & Mai & Jun & Jul & Ago & Set & Out & Nov & Dez & Ano \\
\hline E1 & INMET_Lourdes & 609646 & 7795438 & 915 & 23,83 & 25,43 & 23,65 & 22,32 & 21,64 & 21,25 & 21,39 & 21,63 & 22,45 & 22,26 & 23,45 & 23,88 & 22,77 \\
\hline E2 & INMET_Pampulha & 607921 & 7800869 & 854 & 22,86 & 24,04 & 23,18 & 20,90 & 20,16 & 20,13 & 19,89 & 20,47 & 21,60 & 21,41 & 23,33 & 23,56 & 21,79 \\
\hline E3 & INMET_Ibirite & 595738 & 7786424 & 1199 & 20,75 & 21,94 & 21,04 & 18,98 & 18,31 & 18,28 & 18,01 & 18,33 & 19,22 & 19,13 & 31,40 & 23,61 & 20,75 \\
\hline E5 & FEAM_CAMG & 609887 & 7812486 & 818 & 24,28 & 25,51 & 24,77 & 22,50 & 21,53 & 21,37 & 21,23 & 21,56 & 22,94 & 22,70 & 23,94 & 24,40 & 23,06 \\
\hline E6 & FEAM_Amazonas & 605181 & 7795220 & 880 & 25,37 & 25,41 & 25,53 & 25,73 & 21,82 & 20,50 & 20,45 & 21,08 & 22,14 & 21,84 & 23,14 & 23,88 & 23,07 \\
\hline E7 & FEAM_Piratininga & 598364 & 7787803 & 886 & 25,71 & 24,12 & 26,19 & 19,38 & 20,28 & 20,39 & 20,14 & 20,60 & 21,61 & 21,55 & 22,64 & 23,12 & 22,15 \\
\hline & & & & nédia $=$ & 23,80 & 24,41 & 24,06 & 21,64 & 20,62 & 20,32 & 20,19 & 20,61 & 21,66 & 21,48 & 24,65 & 23,74 & 22,26 \\
\hline
\end{tabular}

Temperatura Média $\left({ }^{\circ} \mathrm{C}\right)$ - Ano 2014 - Regressão Linear

\begin{tabular}{|c|c|c|c|c|c|c|c|c|c|c|c|c|c|c|c|c|c|}
\hline Código & Estações & $\begin{array}{l}\text { Longitude } \\
\text { (x) }\end{array}$ & $\begin{array}{l}\text { Latitude } \\
\text { (y) }\end{array}$ & $\begin{array}{c}\begin{array}{c}\text { Altitude } \\
(\mathrm{m})\end{array} \\
\end{array}$ & Jan & Fev & Mar & Abr & Mai & Jun & Jul & Ago & Set & Out & Nov & Dez & Ano \\
\hline E1 & INMET_Lourdes & 609646 & 7795438 & 915 & 25,78 & 25,23 & 23,99 & 23,31 & 21,67 & 20,99 & 19,94 & 21,69 & 23,86 & 24,19 & 23,80 & 24,89 & 23,28 \\
\hline E2 & INMET_Pampulha & 607921 & 7800869 & 854 & 24,51 & 24,38 & 22,95 & 22,01 & 20,38 & 19,90 & 18,80 & 20,44 & 22,60 & 23,07 & 22,85 & 23,66 & 22,13 \\
\hline E3 & INMET_Ibirite & 595738 & 7786424 & 1199 & 22,14 & 21,95 & 20,81 & 20,27 & 18,57 & 18,05 & 16,80 & 18,61 & 20,27 & 20,65 & 20,52 & 21,35 & 20,00 \\
\hline E4 & INMET_Cercadinho & 608952 & 7790381 & 1200 & 21,91 & 21,40 & 20,27 & 19,77 & 18,24 & 17,48 & 16,34 & 18,31 & 19,95 & 20,16 & 20,21 & 21,25 & 19,61 \\
\hline E5 & FEAM_CAMG & 609887 & 7812486 & 818 & 25,84 & 25,74 & 24,51 & 23,93 & 22,30 & 21,10 & 20,02 & 21,79 & 23,86 & 24,36 & 24,28 & 25,27 & 23,58 \\
\hline E6 & FEAM_Amazonas & 605181 & 7795220 & 880 & 24,62 & 24,96 & 23,22 & 22,09 & 20,56 & 19,91 & 18,88 & 20,46 & 22,74 & 23,21 & 23,06 & 24,13 & 22,32 \\
\hline E7 & FEAM_Piratininga & 598364 & 7787803 & 886 & 24,57 & 24,48 & 23,15 & 22,42 & 20,81 & 20,27 & 19,06 & 20,98 & 22,71 & 23,06 & 23,01 & 23,84 & 22,36 \\
\hline E8 & FEAM_Cidade Industrial & 601329 & 7792728 & 938 & 24,30 & 24,25 & 22,88 & 22,09 & 20,47 & 21,29 & 20,12 & 21,95 & 23,03 & 23,76 & 22,69 & 23,58 & 22,53 \\
\hline \multirow[t]{2}{*}{ E9 } & FEAM_Av_Contorno & 611376 & 7797750 & 840 & 25,47 & 25,22 & 23,76 & 22,86 & 21,31 & 20,72 & 19,69 & 25,17 & 24,11 & 23,78 & 24,79 & 25,56 & 23,54 \\
\hline & & & & édia $=$ & 24,35 & 24,18 & 22,84 & 22,08 & 20,48 & 19,97 & 18,85 & 21,04 & 22,57 & 22,92 & 22,80 & 23,73 & 22,15 \\
\hline
\end{tabular}

Fonte: Elaborado pela autora, 2018.

Após a escolha do melhor interpolador (IDW) para o preenchimento das falhas nos registros da temperatura, foram geradas as tabelas com as médias do mês de abril para cada um dos anos (2013 e 2014) e elaborados os shapes dos pontos amostrais no ArcGis $10.3 \mathrm{com}$ os valores de cada variável. Adotou-se a classificação pelo método jenks natural breaks em quatro classes. Após essa etapa, foram elaborados os mapas das figuras 7 e 8.

Foram utilizados os registros da estação meteorológica convencional do INMET (Lourdes) para os horários de 00h, 12h e 18h UTC dos anos de 2013 e 2014, para elaborar os gráficos de temperatura do ar, precipitação e umidade relativa, velocidade e direção dos ventos e dos dados da estação meteorológica da FEAM (CAMG), para o mesmo período e horário, na confecção dos gráficos de PM10 e CO (FIG. 4 e 5).

Com a finalidade de buscar uma correlação entre as mudanças na condição de tempo e os picos registrados na ocorrência das doenças respiratórias, foi realizada a caracterização dos tipos de tempo e a análise diária da sucessão dos estados atmosféricos observada no período amostral, tendo como instrumental teórico-metodológico os gráficos de análise rítmica (MONTEIRO, 1971). Utilizaram-se cartas sinóticas disponibilizadas pelo Centro de Hidrografia da Marinha do Brasil e imagens de satélite cujo download foi realizado na Divisão de Satélites e Sistemas Ambientais, vinculado ao Instituto Nacional de Pesquisas Espaciais (INPE). Os horários analisados foram de $00 \mathrm{H}$ e 12H UTC para os 30 dias do mês de abril dos anos de 2013 e 2014 (FIG. 4 e 5).

Outra variável que foi levada em consideração nesse estudo foi a densidade demográfica da população de 0 a 5 anos, calculada através dos dados do Censo Demográfico do IBGE (2010). Essa informação foi exportada no 
formato raster para ser utilizada no método de análise hierárquica de pesos, descrita a seguir.

Com objetivo de reforçar as análises de correlação espacial das variáveis ambientais e a ocorrência de doenças respiratórias, adotou-se a Análise Hierárquica de Pesos (AHP). A AHP é um método de Análise Multicritério (AMC) utilizado para auxiliar as tomadas de decisões. Esse método é baseado em julgamentos de profissionais ou pesquisadores relacionados com a temática abordada no qual se propõe um conjunto de variáveis ou indicadores que devem ser comparados par a par e indicado o grau de importância de uma em relação à outra.

A análise multicritério tem sido usada com frequência entre os pesquisadores que adotam os SIG's em que o objetivo é a construção de modelos descritivos ou preditivos do território, em diferentes escalas e para diferentes aplicações, viabilizando a integração de variáveis espaciais (Moura et al. ,2016).

Foi enviado um questionário, via Google Drive, para os profissionais da área da saúde visando entender quais das variáveis acima têm maior peso no agravo das doenças respiratórias. Foi solicitado aos participantes que classificassem com valores de 1 a 9, onde um tem menor importância e nove extrema importância. As variáveis eleitas para esse estudo foram: umidade relativa $(\%)$, temperatura $\left({ }^{\circ} \mathrm{C}\right)$, amplitude térmica mensal $\left({ }^{\circ} \mathrm{C}\right)$, partículas inaláveis - PM10 $(<10 \mu \mathrm{m} / \mathrm{m} 3)$, monóxido de carbono - CO $(\mathrm{ppm})$, densidade populacional (hab/ $\mathrm{Km}^{2}$ ) e distrito sanitário (9). Ressalta-se que o peso 0 (zero) não foi adotado como mínimo porque se considera que nenhuma das variáveis é desprezível, assim como o peso 10, evidenciando que nenhuma das variáveis possui importância tão grande sobre a outra. Ao final do questionário foram inseridas as seguintes questões:

1)Retiraria alguma das variáveis analisadas? Por quê?

2)Existe alguma variável que julgue importante e não foi contemplada?

3)Gostaria de fazer alguma consideração ou comentário que ache pertinente em relação ao tema pesquisado?

O resultado foi considerado satisfatório para o método adotado, pois dos 12 profissionais da área da saúde convidados, sete enviaram suas respostas e comentários. De posse das respostas, foram eleitos três cenários: "cenário geral", "cenário especialista 1" e "cenário especialista 2". Ressalta-se que o valor da relação de consistência (consistency ratio - CR) foi próximo a zero ou zero, reforçando a confiabilidade da modelagem. Com os rasters das variáveis finalizados, foi gerada a AHP através do software ArcGis 10.3 que rodou duas mil (2.000) combinações possíveis entre eles.

A partir dessa etapa foi possível a espacialização dos dados e produção de combinações cartográficas capazes de contribuir para uma melhor compreensão dos lugares de ocorrência das doenças e suas intensidades.

\section{RESULTADOS E DISCUSSÕES}

A análise dos dados relacionados à ocorrência de doenças respiratórias em crianças de 0 a 5 anos no município de Belo Horizonte, mostra que os meses de março, abril, agosto, setembro e outubro são os meses em que se intensifica 
o número de atendimentos em unidades de saúde. Porém o pico dos registros ocorreu em abril para os dois anos analisados (FIG.3). Em abril de 2013 o número de atendimentos correspondeu a $15 \%$ ( 8.290 atendimentos) do total (56.566 atendimentos) e em 2014 correspondeu a 13\% (9.568 atendimentos) do total (72.977 atendimentos).

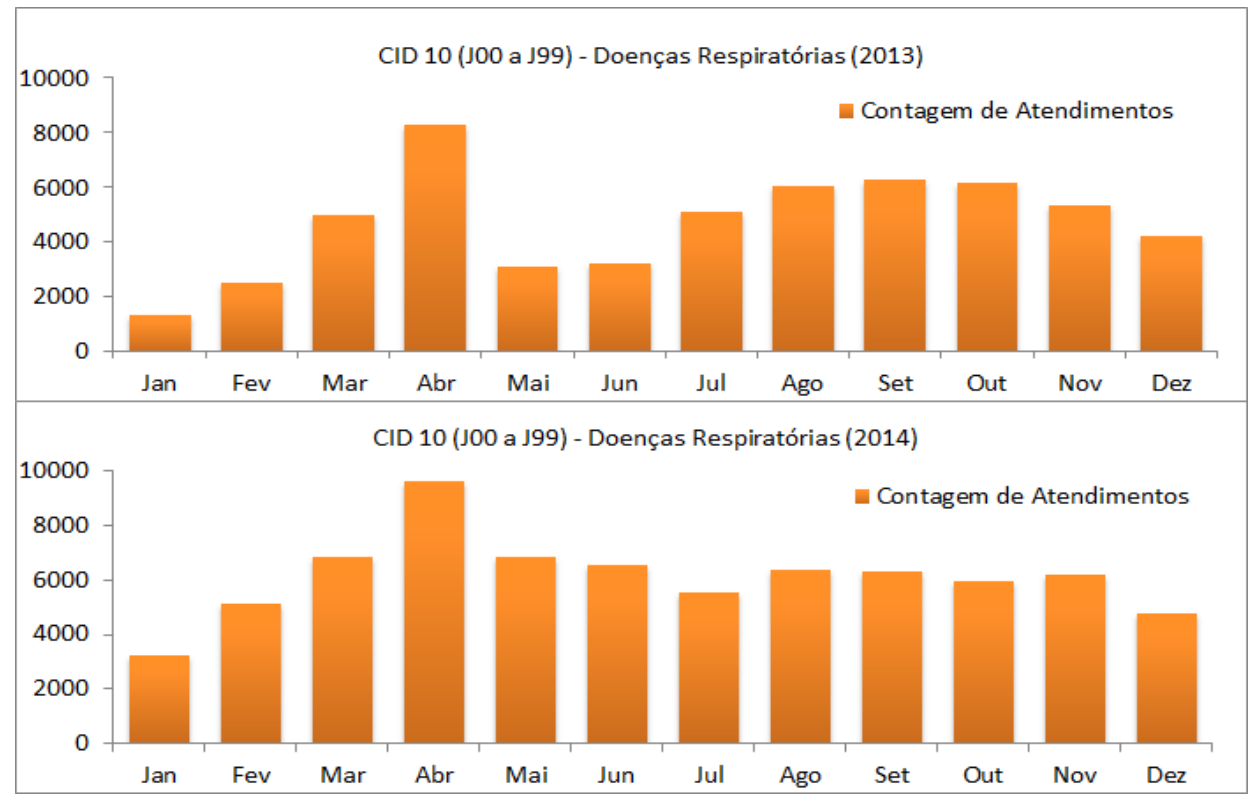

Figura 2 - Número de ocorrência de doenças respiratórias registradas no município de Belo Horizonte em abril de 2013 e 2014.

A partir da análise rítimica do mês de abril para os anos de 2013 e 2014, foi possível verificar que a redução no volume diário de precipitação proporcionou um aumento gradativo na quantidade de material particulado MP10 e CO na atmosfera belo-horizontina. Nesse período, foram registradas elevadas amplitudes térmicas diárias em função da atuação do Anticiclone Subtropical do Atlântico Sul (ASAS). A permanência desse sistema por vários dias na região faz com que o mesmo adquira características de continentalização, elevando a temperatura e reduzindo ainda mais a umidade relativa do ar.

Segundo Assis et al. (2013), as condições atmosféricas observadas no município de Belo Horizonte são controladas pela atuação de sistemas sinóticos estáveis, tais como o ASAS e a Massa Polar Atlântica (mPa), e instáveis, como Frentes Frias (FF), Frentes Estacionárias (FE), Linhas de Instabilidade (LI), Zona de Convergência de Umidade (ZCOU) e a Zona de Convergência do Atlântico Sul (ZCAS). Entretanto, os totais pluviométricos diários mais significativos ocorreram durante a atuação da ZCAS e das LI. Vale destacar que as Instabilidades Convectivas Locais (ICL) têm uma participação importante nas chuvas torrenciais nos meses de dezembro, janeiro, fevereiro e março.

No mês de abril de 2013 foi possível identificar o maior número de resgistros de atendimentos por doenças respiratórias (FIG.4). Esses coincidiram com os dias de atuação de frentes frias e de linhas de instabilidade sucedidos pela chegada da massa polar atlântica. A mPa provocou quedas abruptas na temperatura e ausência de precipitação. Ao longo da $3^{a}$ semana do mês em 
questão, percebeu-se a tropicalização da mPa acompanhada de um aumento na amplitude térmica, queda na umidade relativa do ar e do acúmulo de material particulado na atmosfera belo-horizontina.

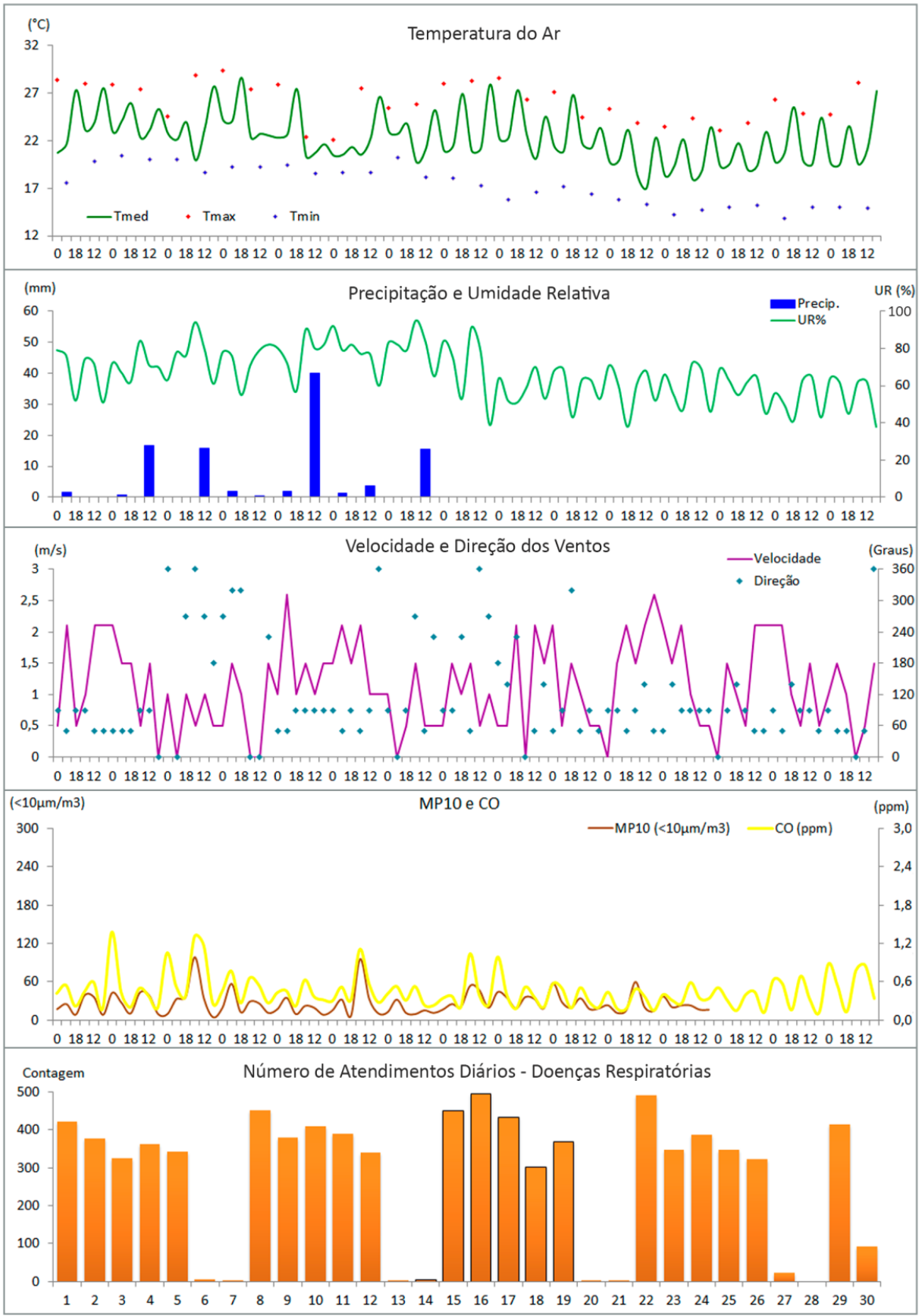

Sistemas Atmosféricos
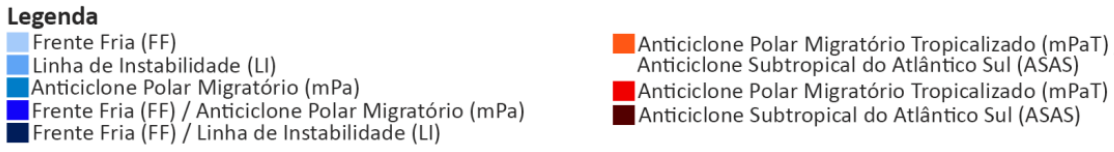

Figura 3 - Análise rítmica do mês de abril de 2013. 
Para o mês de abril de 2014 foi possível indentificar, principalmente na $2^{a}$ semana, o maior número de resgistros de atendimentos por doenças respiratórias(FIG.5). A atuação da mPat por vários dias no mês analisado, proporcionou elevada amplitude térmica elevada e queda significativa na umidade relativa do ar. Na terceira semana, iniciou a atuação do ASAS com registros de altas quantidades de material particulado. Após a atuação desse sistema, verificou a chegada de uma Frente Fria na Região Metropolitana de Belo Horizonte (FF: a temperatura e a umidade diminuem, a pressão sobe e o vento muda de direção) proporcionando precipitações de baixa intensidade. 


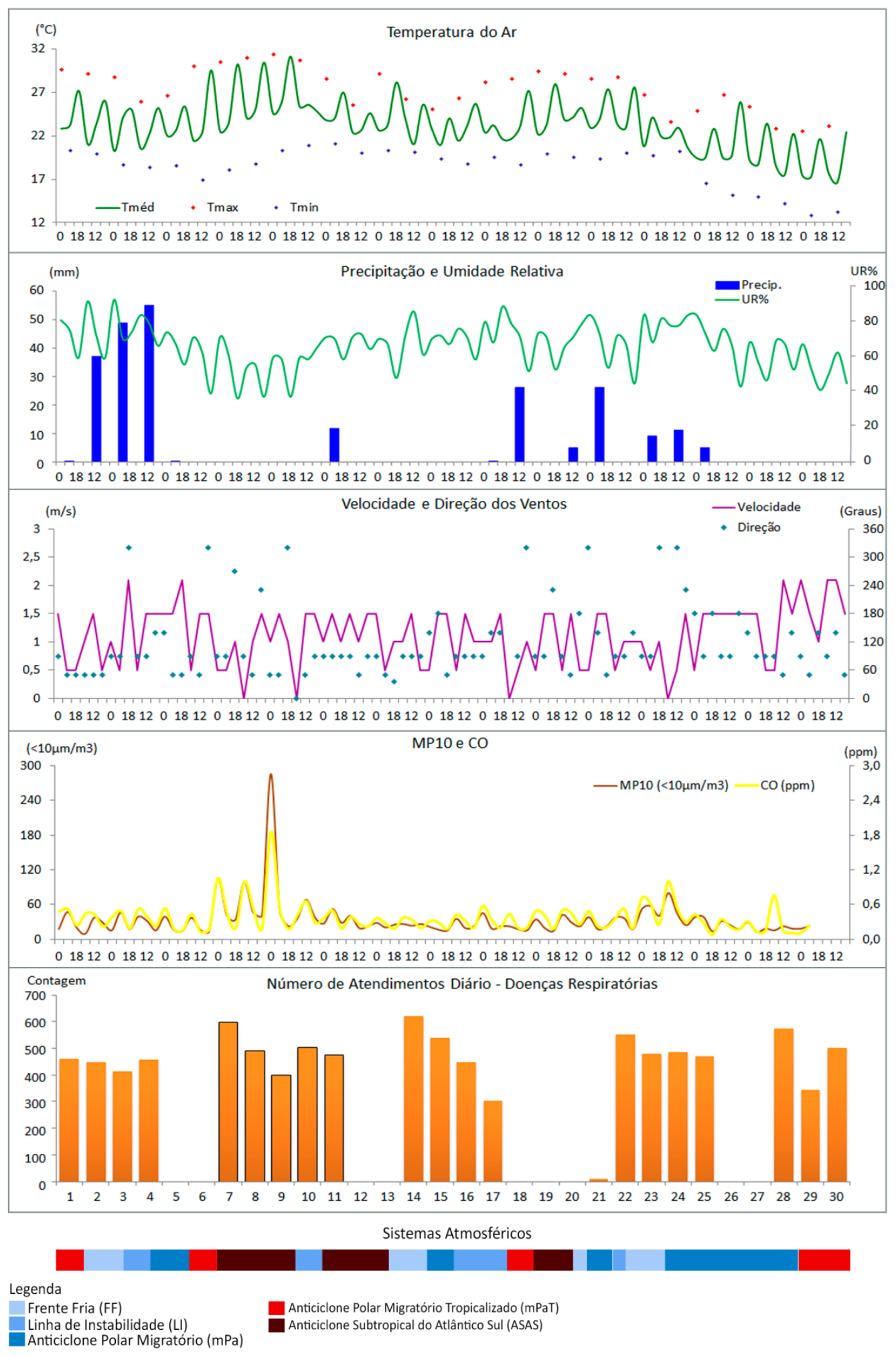

Figura 4 - Análise rítmica do mês de abril de 2014. 
Ao lidar com as variáveis climáticas, percebe-se que não há limites rígidos nas dimensões espaciais. Para tanto, são utilizados balizadores com o objetivo de auxiliar a espacialização do fenômeno estudado. Como ferramenta de apoio, os sistemas de informações geográficas (SIG's) possibilitam uma análise espaço-temporal importante em estudos como este.

De posse dos resultados da modelagem, realizadas no software ArcGIS 10.3 por meio da ferramenta AHP, foi possível identificar no município as áreas com maior (vermelho) e menor (verde) potencial de ocorrência de doenças respiratórias. As figuras 7 e 8 mostram a representação da análise multicritério para os anos de 2013 e 2014. Essa análise levou em consideração todas as variáveis abordadas na AHP, descrita nos procedimentos metodológicos, a partir dos pesos de cada um dos três cenários: "cenário geral", "cenário especialista 1" e "cenário especialista 2 " conforme tabela 2 .

Tabela 2 - Matrizes com os resultados da AHP para os três cenários adotados.

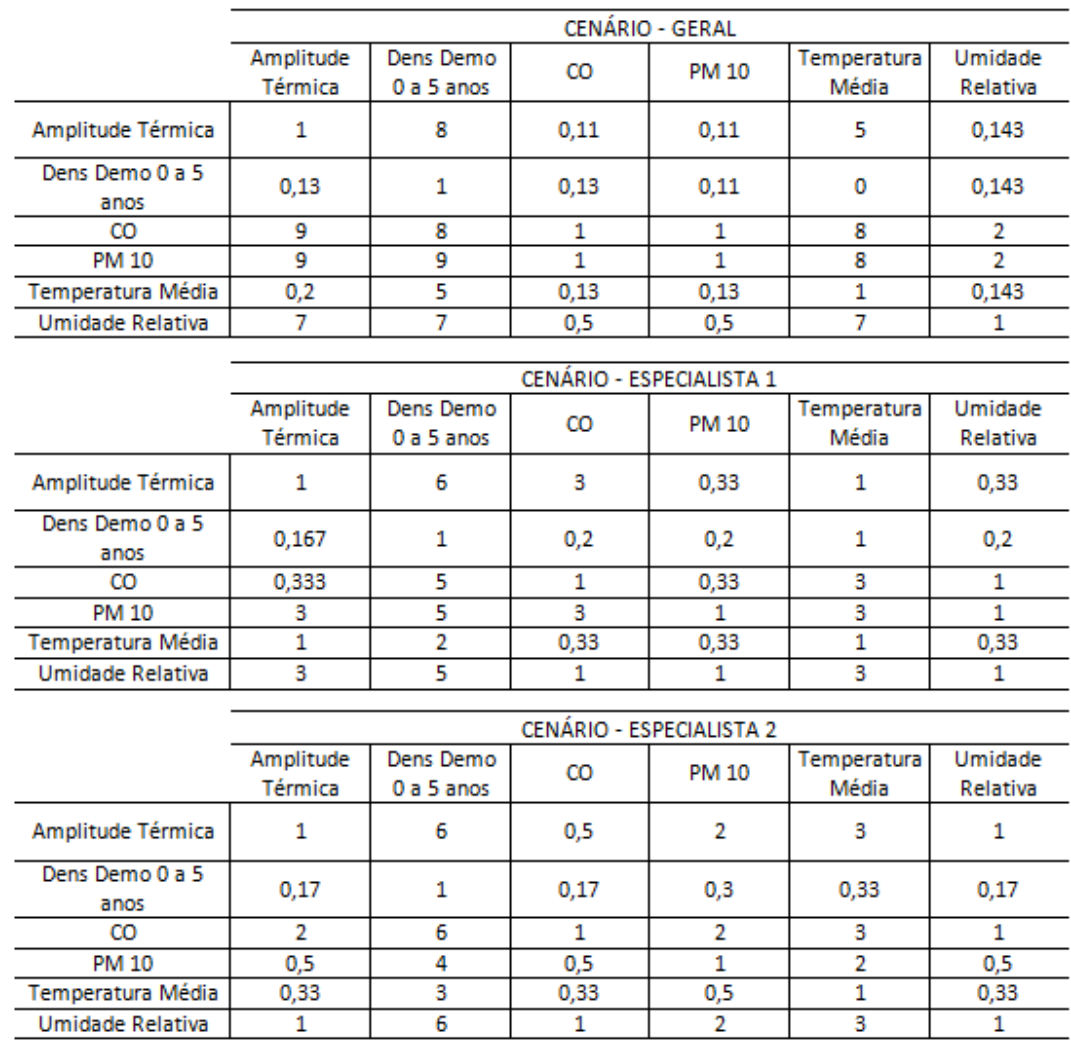

\begin{tabular}{|c|c|}
\hline \multicolumn{2}{|l|}{ Pesos AHP } \\
\hline Amplitude Térmica & 7,746 \\
\hline $\begin{array}{c}\text { Dens Demo } 0 \text { a } 5 \\
\text { anos }\end{array}$ & 2,085 \\
\hline $\mathrm{CO}$ & 32,265 \\
\hline PM 10 & 32,564 \\
\hline Temperatura Media & 3,872 \\
\hline Umidade Relativa & 21,469 \\
\hline $\mathrm{CR}$ & Zero \\
\hline \multicolumn{2}{|l|}{ Pesos AHP } \\
\hline Amplitude Térmica & 17,107 \\
\hline $\begin{array}{c}\text { Dens Demo } 0 \text { a } 5 \\
\text { anos }\end{array}$ & 3,797 \\
\hline $\mathrm{CO}$ & 15,321 \\
\hline PM 10 & 29,904 \\
\hline Temperatura Media & 8,611 \\
\hline Umidade Relativa & 25,259 \\
\hline$C R$ & 0,096 \\
\hline \multicolumn{2}{|l|}{ Pesos AHP } \\
\hline Amplitude Térmica & 22,09 \\
\hline $\begin{array}{c}\text { Dens Demo } 0 \text { a } 5 \\
\text { anos }\end{array}$ & 3,652 \\
\hline $\mathrm{CO}$ & 28,021 \\
\hline PM 10 & 13,483 \\
\hline Temperatura Média & 8,361 \\
\hline Umidade Relativa & 24,392 \\
\hline $\mathrm{CR}$ & 0,014 \\
\hline
\end{tabular}

Fonte: Elaborado pela autora, 2018.

Nas figuras 7 e 8 foi realizado um mosaico de mapas considerando as variáveis amplitude térmica, temperatura média, umidade relativa, CO, PM10 e a análise multicritério para os anos de 2013 e 2014.

Tomando como base as áreas de elevado potencial de ocorrência de doenças respiratórias (cores quentes), é possível realizar associações espaciais quanto à ocorrência e distribuição do fenômeno (porção centro-oeste e sudoeste do município), correlacionando-as a áreas de maior amplitude térmica, menores temperaturas e baixa umidade relativa. Com relação aos materiais particulados, 
percebe-se que os mesmos têm influência significativa na ocorrência das doenças respiratórias no município de Belo Horizonte. Para as áreas com menor potencial de ocorrência de doenças respiratórias (tons de verde) observa-se que a variável umidade relativa torna-se um indicador preponderante no modelo gerado, pois as áreas de umidade relativa acima de $70 \%$ coincidem geograficamente com as zonas de menor potencial de ocorrência.

Abril de 2013

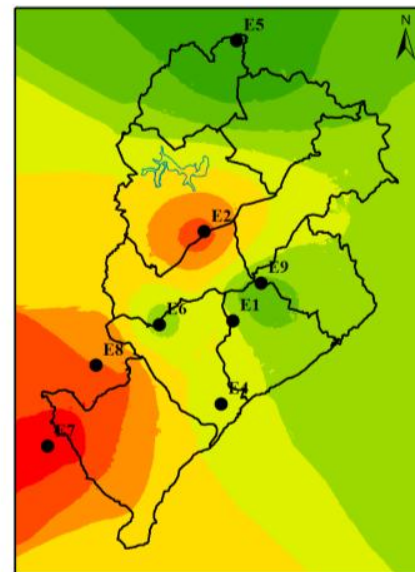

Análise Multicritério - Potencial de Ocorrência de Doenças Respiratórias 0 - $0,13 \square 0,50-0,62$ $\square 0,14-0,27 \square 0,63-0,74$ $\square 0,28-0,36 \square 0,75-0,87$ $\square 0,37$ - 0,49 $\square, 88$ - 0,99

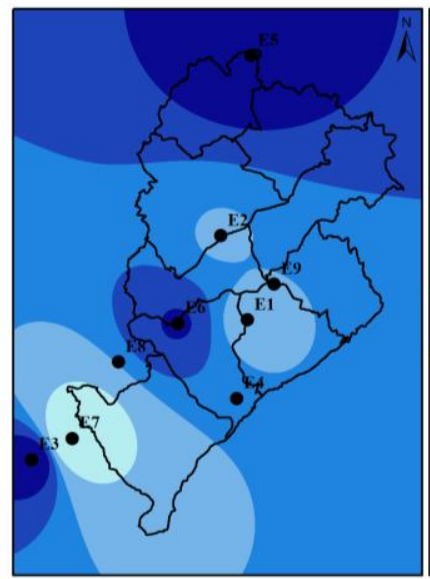

Umidade Relativa (\%)

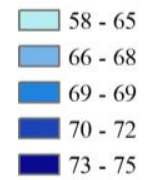

$\mathrm{U} F m \mathrm{G}$

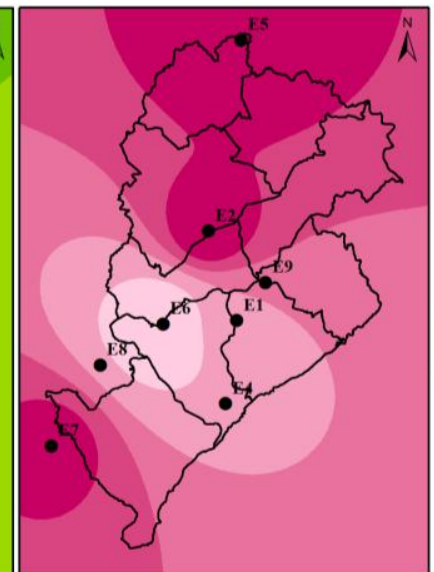

Amplitude Térmica $\left({ }^{\circ} \mathrm{C}\right)$

$\square 2,8-8,1$

$\square 8,2-10,9$

$\square 11-12,5$

$12,6-14$

$14,1-16,2$

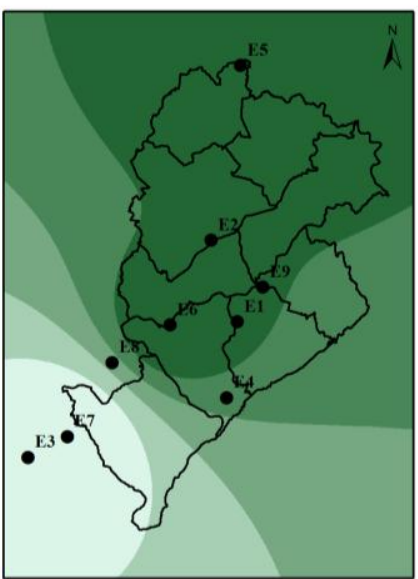

Monóxido de Carbono (ppm)

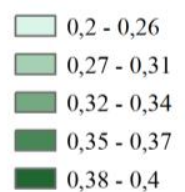

$0,38-0,4$

Programa de Pós-Graduação em Geografia Área de Concentração: Análise Ambiental Elaboração: Denise Marques Sales Data: 14/08/2018 Datum: SIRGAS 2000 Projeção: UTM Fonte: FEAM (2017), Prodabel (2017), INMET (2018), IBGE(2010)

Figura 5 - Mosaico da interpolação dos elementos climáticos e análise multicritério para abril de 2013. 
Abril de 2014

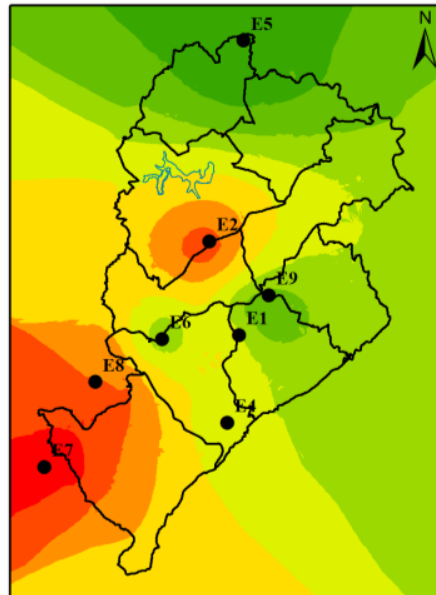

Análise Multicritério - Potencial de Ocorrência de Doenças Respiratórias 0 - 0,13 $\square 0,50-0,62$ $\square 0,14-0,27 \square 0,63-0,74$ $\square 0,28-0,36 \square 0,75-0,87$ $\square 0,37$ - 0,49 $\square 0,88$ - 0,99

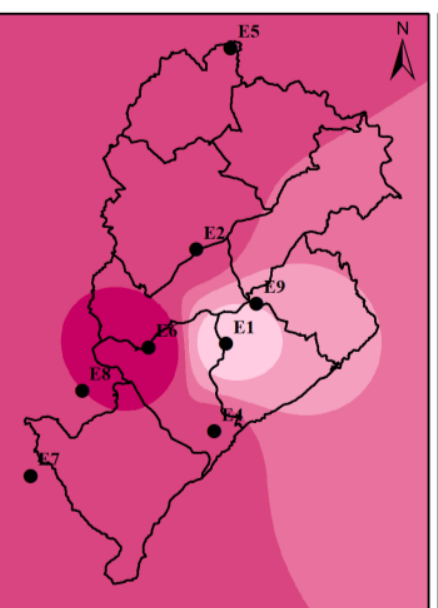

Amplitude Térmica $\left({ }^{\circ} \mathrm{C}\right)$

$\square$ 9,4 - 13,5

$\square 13,6-15,9$

$\square 16-17,1$

$17,2-18,8$

$18,9-22,5$

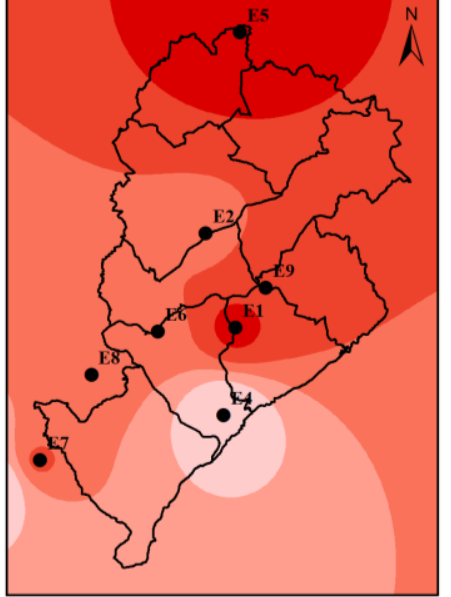

Temperatura Média $\left({ }^{\circ} \mathrm{C}\right)$

$\square 19,8-21,2$

$\square 21,3-21,8$

$\square 21,9-22,3$

$22,4-22,9$

$23-23,9$

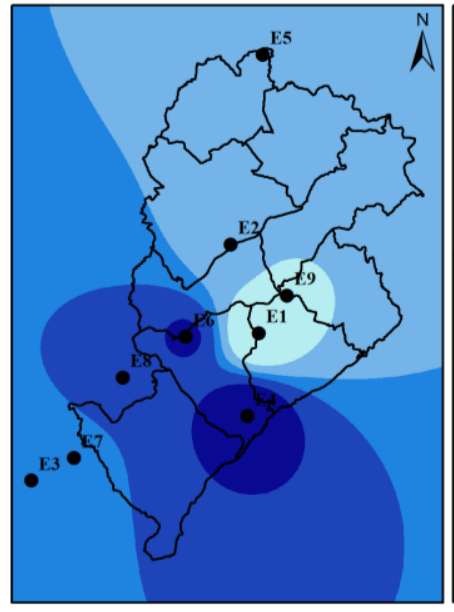

Umidade Relativa (\%)

$\square 68$ - 71

$\square 72-72$

$73-73$

$74-74$

$75-77$

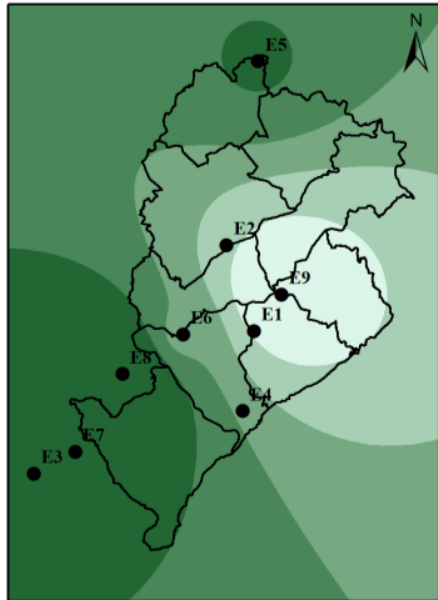

Monóxido de Carbono (ppm)

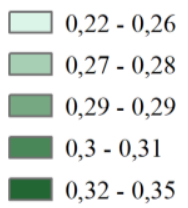

$\square 0,22-0,26$

$0,32-0,35$
Distritos Sanitários (SUS)

- Estações Climatológicas (INMET/FEAM)

\section{$\mathrm{U} F m \mathrm{G}$}

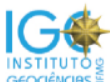

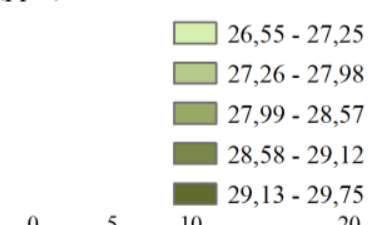

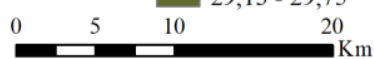

Programa de Pós-Graduação em Geografia Área de Concentração: Análise Ambiental Elaboração: Denise Marques Sales Data: 14/08/2018 Datum: SIRGAS 2000 Projeção: UTM Fonte: FEAM (2017), Prodabel (2017), INMET (2018), IBGE(2010)

Figura 6 - Mosaico da interpolação dos elementos climáticos e análise multicritério para abril de 2014.

A partir desse conjunto de análises (AHP e ritmo climático) foi possível perceber uma predominância do impacto negativo dos materiais particulados à 
saúde da população estudada. Além disso, percebeu-se que a amplitude térmica e a umidade relativa têm papeis significativos nos agravos a saúde respiratória da população belo-horizontina e funcionam como gatilho para o adoecimento das crianças de 0 a 5 anos.

Outra ressalva importante, descrita pelos profissionais da saúde que responderam ao questionário, foi a necessidade de inclusão da condição social e/ou da vulnerabilidade das populações no território urbano analisado. Esse fator é tratado de maneira incisiva nos documentos da OMS. Entretanto, nesse estudo não foi possível incluir essas variáveis (não havia tido acesso ao dado em formato vetor ou raster). Porém, o município de Belo Horizonte possui o Índice de Vulnerabilidade da Saúde (IVS) elaborado em 2013 pela prefeitura. O IVS leva em consideração as dimensões saneamento básico e condições socioeconômicas divididas em oito indicadores. Esse índice categorizou o município em quatro tipos na escala espacial de setores censitários, a saber: baixo risco, médio risco, elevado risco e muito elevado risco.

Faz-se necessário ressaltar que os resultados ficariam mais robustos e ajustados se houvesse a disponibilidade de um banco de dados meteorológicos com uma maior abrangência espacial e temporal. O ideal seria realizar um estudo que abarcasse uma série temporal de 10 anos, ou mais, para ter resultados mais conclusivos. Cabe reforçar que existe no Brasil um déficit de manutenção e atualização de bancos de dados e principalmente de tornar pública a disponibilização dessas informações. Sejam elas relacionadas aos registros climáticos, ambientais e urbanos, o que dificulta boa parte dos trabalhos que lidam com esses dados e buscam sua integração para estudos e pesquisas.

\section{CONCLUSÕES}

A climatologia urbana tem trazido grandes contribuições para avaliação da qualidade de vida, dando a devida atenção para os aspectos que condicionam o conforto térmico e a saúde das populações. Para tanto, é necessário estudar os cenários urbanos, perceber sua evolução histórica, avaliar aspectos ambientais, socioeconômicos, elencar os riscos, tipos de vulnerabilidade e entender os tipos de acesso à saúde disponível em cada contexto, averiguando a existência de instrumentos e protocolos para evitar agravos à saúde humana.

As interrelações entre saúde e os aspectos ambientais em áreas urbanas não podem serem tratadas de forma isolada. É preciso correlacionar uma gama de fatores, sejam eles físicos, sociais, econômicos, políticos, culturais e epidemiológicos para a redução do risco às doenças respiratórias, conforme sugerem os documentos produzidos pela Organização Mundial da Saúde.

Nesse estudo foram identificados os meses de maior pico de ocorrência de doenças respiratórias, elegendo-se o mês de abril como foco das análises. Foram realizadas as etapas de seleção das variáveis climáticas, preenchimento dos dados faltantes a partir de interpolação e regressão linear, e a realização da análise rítmica. Em seguida a análise hierárquica de pesos, que incluiu além das variáveis climáticas a densidade demográfica por setor censitário da população de 0 a 5 anos, chegando à uma primeira espacialização dos dados e produção de combinações cartográficas interessantes para identificação das interações entre clima e saúde urbana no município. 
O estudo indicou que a partir do mês abril o volume diário de precipitação sofre redução e verifica-se um aumento gradativo na quantidade de particulados e CO na atmosfera belo-horizontina, gerando um acumulo de poluentes que conforme Duchiade (1992) e Santos (2007) causam efeitos nocivos sobre as vias respiratórias agravando os sintomas em indivíduos vulneráveis.

As análises apontam para um período em que são registradas elevadas amplitudes térmicas diárias em função da participação de sistemas atmosféricos estáveis, principalmente do Anticiclone Subtropical do Atlântico Sul e da Massa Polar Atlântica. A atuação desses sistemas em Belo Horizonte proporcionam diferenças bruscas de temperatura que podem influenciar no desconforto da população e propiciar a multiplicação de micro-organismos, como vírus e bactérias, causando agravos à saúde.

Os resultados demonstraram a importância do comportamento temporal das variáveis meteorológicas e do ritmo climático no agravo das doenças respiratórias. A partir desses resultados foi possível observar que a baixa umidade relativa do ar, a redução na precipitação, a alta amplitude térmica e o elevado grau de materiais particulados na atmosfera belo-horizontina ao longo dos meses de abril (2013 e 2014), proporcionaram aumentos significativos na ocorrência das doenças do aparelho respiratório. Resultados esses que coadunam com a declaração da OPAS (2008) que o clima, entre outros fatores, pode suscitar a manifestação de determinadas doenças à saúde, neste caso em escala local.

É importante ressaltar que os resultados relatados nesse estudo não esgotam a possibilidade de conclusões acerca das interrelações entre elementos climáticos e doenças respiratórias. Esse artigo corresponde a parte de uma pesquisa que está em andamento em que novas etapas metodológicas estão sendo aplicadas e contarão com análises de correlação espacial utilizando como unidade de análise o setor censitário. Serão feitas correlações entre o resultado da AMC (análise de aptidão para a ocorrência de doenças) com a ocorrência efetiva de doenças respiratórias; correlações entre o IVS e a ocorrência efetiva de doenças respiratórias; e por fim, entre IVS e AMC, com a finalidade de enriquecer as análises. Utilizando como metodologia complementar o Índice de Moral Local e Global e a auto correlação entre as variáveis selecionadas.

Propõe-se, ainda, incentivar parcerias entre a academia e os setores públicos e privados, para que interajam e tenham uma linguagem comum para elaborarem diagnósticos e propor novas alternativas de intervenção.

\section{REFERÊNCIAS BIBLIOGRÁFICAS}

ANTUNES, A. F.; BÁRBARA, C.; GOMES, E. M. Programa nacional para as doenças respiratórias (PNDR) 2012 - 2016. Direcção-Geral da Saúde, 2a edição, p. $1-18,2013$.

ASSIS, W. L.; PARIZZI, M. G.; RIBEIRO, S. M.; Identificação dos sistemas atmosféricos responsáveis por inundações e deslizamentos no município de Belo Horizonte: análise dos períodos chuvosos de 2011-2012 e 2012-2013. Revista Geografias, Belo Horizonte, v. 9, n. 2, p.38-55, 2013.

BELO HORIZONTE. Secretaria Municipal de Saúde (SMSA). Assessoria de Tecnologia e Informação em Saúde (ASTIS). 2017. 
BELO HORIZONTE. Secretaria Municipal de Saúde (SMSA). Índice de Vulnerabilidade da Saúde (IVS) - 2013.

BRASIL. Ministério da Saúde. Doenças respiratórias crônicas. Brasília: Ministério da Saúde. Cadernos de Atenção Básica, n. 25 (Série A. Normas e Manuais Técnicos). 2010.

DUCHIADE, M. P. Poluição do ar e doenças respiratórias: uma revisão. Cadernos de Saúde Pública, v. 8, n. 3, p. 311-330, 1992.

INSTITUTO NACIONAL DE METEOROLOGIA (INMET). 2018.

INSTITUTO BRASILEIRO DE GEOGRAFIA E ESTATÍSTICA (IBGE). Censo Demográfico 2010.

JANKOWSKI, P. L. Análise de sensibilidade espacial para avaliação de aptidão da terra: SASE. In: MOURA et al (org.). Tecnologias de geoinformação para representear e planejar o território urbano. Editora Interciência, Rio de Janeiro, p. 115 - 132, 2016.

MINAS GERAIS. Fundação Estadual do Meio Ambiente (FEAM). 2017.

MONTEIRO, C. A. F. Análise rítmica em climatologia: problemas da atualidade climática em São Paulo e achegas para um programa de trabalho. São Paulo, Série Climatologia Dinâmica I, USP-IG, p. 21, 1971.

MOURA, A. C. M.; JANKOWSKI, P. Contribuições aos estudos de análises de incertezas como complementação às análises multicritérios - "Sensitivity analysis to suitability evaluation". Revista Brasileira de Cartografia, v. 68/4, p. 665-684, 2016.

OPAS - Organização Pan-Americana da Saúde. Mudanças climáticas e ambientais e seus efeitos na saúde: cenários e incertezas para o Brasil. Brasília (DF), 2008.

PERIN, E. B.; VIANNA, L. F. N.; RICCE, W. S.; MASSIGNAM, A. M.; PANDOLFO, C.. Interpolação das variáveis climáticas temperatura do ar e precipitação: Revisão dos Métodos Mais Eficientes. Revista Geografia, Rio Claro v. 40, n. 2, p. 269-289, 2015.

PREFEITURA DE BELO HORIZONTE. Empresa de Informática e Informação do Município de Belo Horizonte (PRODABEL). Base Cadastral PRODABEL, 2017.

SANTOS, A. R.; RIBEIRO, C. A. A. S.; SEDIYAMA, G. C.; PELUZIO, J. B. E.; PEZZODANE, J. E. M.; BRAGANÇA, R. Espacialização de Dados Meteorológicos no ArcGIS 10 . 3 Passo a Passo. CAUFES. 1. Ed. v. 1. p 1 - 64, 2015.

SANTOS, M. O retorno do território. In: SANTOS, M.; SOUZA, M. A. A. DE.; SILVEIRA, M. L. Território: globalização e fragmentação. São Paulo, p. 15-20, 1998.

SANTOS, U. DE P. Poluição, aquecimento global e repercussões na saúde. Revista da Associação Médica Brasileira, v. 53, n. 3, p. 193-194, 2007.

WHO - World Health Organization. Air quality guidelines for particulate matter, ozone, nitrogen dioxide and sulfur dioxide: Global update 2005. Disponível em: http://www.who.int/phe/health_topics/outdoorair/outdoorair_aqg/en/ Acesso em: 25 jun 2018. 
WHO - World Health Organization. Global Alliance against chronic Respiratory Diseases. World Health Organization. Disponível em: <http://www.who.int/gard/en/>. Acesso em: 05 jul 2018.

WHO - World Health Organization. Global surveillance, prevention and control of chronic respiratory diseases: a comprehensive approach. Geneva, World Health Organization, $2007 . \quad$ Disponível em: <http://www.who.int/gard/publications/GARD_Manual/en/index.html>. Acesso em: 27 jun 2018.

WHO - World Health Organization. Preventing chronic diseases, a vital investment. Geneva, World Health Organization, 2005. Disponível em: <http://www.who.int/chp/chronic_disease_report/en/index.html>. Acesso em: 25 jun 2018. 